\title{
DOES THE PLATELET CONCENTRATION IN PLATELET RICH PLASMA INFLUENCE THE OUTCOMES OF PRIMARY KNEE OSTEOARTHRITIS?
}

\author{
Brang Mai, Maung Mg Htwe, Than Win, Zaw Min Han, Aung Myo
}

Department of Orthopaedic Surgery, University of Medicine, Mandalay, Myanmar

\begin{abstract}
Background: Growth factors in platelets have been extensively studied and were reported to be used to stimulate cartilage regeneration in osteoarthritis $(\mathrm{OA})$.

Objective: This study aimed to observe the influence of platelet concentration in platelet rich plasma (PRP) on the outcomes of primary knee OA.

Methods: Eighty-nine patients undergoing PRP injection in unilateral primary knee OA were assessed using the Western Ontario and McMaster Universities Arthritis Index (WOMAC) questionnaire and visual analog scale (VAS) before intervention at 3 weeks, 3 months, 6 months and 12 months after treatment. A small aliquot of PRP was sent for bacteriologic examination and evaluation of the platelet count. A student t-test was conducted to compare WOMAC and VAS score among patients before PRP injection (baseline) and at each follow-up. The platelet count and their influence on outcomes were also analyzed using Pearson`s correlation coefficient.
\end{abstract}

Results: Statistically significant differences were observed in the WOMAC score between baseline $(\mathrm{M}=47.08, \mathrm{SD}=8.50)$ and 3 weeks $(\mathrm{M}=20.37, \mathrm{SD}=10.09, p<0.001), 3$ months $(\mathrm{M}=23.24, \mathrm{SD}=11.39$, $p<0.001), 6$ months $(\mathrm{M}=29.89, \mathrm{SD}=14.95, p<0.001)$, and final follow-up at 12 months $(\mathrm{M}=27.78$, $\mathrm{SD}=16.56, p<0.001)$. Also a significant difference was observed in VAS between baseline $(\mathrm{M}=69.02$, $\mathrm{SD}=9.58)$ and 3 weeks $(\mathrm{M}=36.23, \mathrm{SD}=15.72, p<0.001), 3$ months $(\mathrm{M}=37.04, \mathrm{SD}=17.30, p<0.001)$, 6 months $(\mathrm{M}=42.58, \mathrm{SD}=22.15, p<0.001)$ and 12 months $(\mathrm{M}=39.15, \mathrm{SD}=23.96, p<0.001)$. The mean

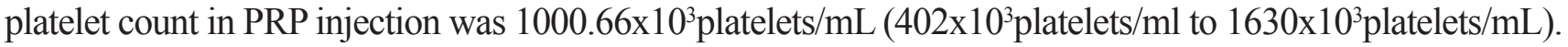
Positive correlations were discovered between the concentration of the platelet and the mean improvement WOMAC scores and VAS at 3 weeks $(\mathrm{r}=0.31, \mathrm{r}=0.40), 3$ months $(\mathrm{r}=0.10, \mathrm{r}=0.23)$, 6 months $(\mathrm{r}=0.08, \mathrm{r}=0.30)$ and 12 months after intervention $(\mathrm{r}=0.12, \mathrm{r}=0.23)$, respectively.

Conclusion: Higher concentrations of platelets in the PRP had a better effect on outcomes of primary knee OA especially at three weeks after injection.

Keywords: Concentration of platelets, Platelet rich plasma, Primary knee OA

J Southeast Asian Med Res 2021: 5(1): 1-10

http://www.jseamed.org

Correspondence to: Brang Mai, Department of Orthopaedic Surgery, University of Medicine, Mandalay, Myanmar

E-mail: dr.brangmai@gmail.com

Received: 11 January 2021

Revised: 30 April 2021

Accepted: 10 May 2021 


\section{Introduction}

Platelet rich plasma (PRP) is orthobiologic and several studies have reinforced that PRP has positive clinical results in treating osteoarthritis $(\mathrm{OA}){ }^{(1-8)}$ Platelet rich plasma is the volume of concentrated platelets extracted from plasma usually 3 to 5 times above baseline. The platelets contain alpha granules that are rich in several growth factors such as platelet derived growth factor (PDGF), transforming growth factors- $\beta$, insulin like growth factors, vascular endothelial growth factor, epidermal growth factors and the release of these biologically active proteins from platelets is part of the natural healing process and tissue regeneration. ${ }^{(9-12)}$ Growth factors in platelets have been extensively studied in OA and have been reported to be used as an attractive method to stimulate cartilage regeneration in $\mathrm{OA} .^{(11,13)}$

The concentration of growth factors depends on the platelet count ${ }^{(14-17)}$ and may affect the outcomes of the disease. Few studies have estimated the growth factors involved ${ }^{(18,19)}$ as well as platelet count in PRP which is used to inject into the knee joint. ${ }^{(6,18,19)}$ The platelet concentration in PRP is essential for fracture healing and the optimal biological effects on pain relief and functional recovery are associated with platelet concentrations of approximately $1,000 \times 10^{3}$ platelet $/ \mathrm{mL}$. ${ }^{(20,21)}$ However, to our knowledge no report have determined the number of platelets required for effective regenerative therapy as well as the influence of platelet concentration in PRP on the outcomes of primary knee OA. This raises the question whether an increased platelet concentration in PRP has better efficacy in knee OA. This study observed the influence of platelet concentration in PRP on the outcomes of knee OA.

\section{Methods}

The study was approved by the Academic Committee of the University of Medicine Mandalay, Myanmar and informed consent from all patients was obtained. The following diagnostic criteria were applied: patients with primary unilateral knee OA with the history of chronic knee pain lasting at least 12 months and the radiologic signs of the knee OA grades 1, 2 or 3 according to Kellgren \& Lawrence Radiographic Knee OA classification (K\&L). Exclusion criteria were OA secondary to inflammatory diseases, patients with generalized OA, bilateral OA knee, advanced staged of knee OA, patients receiving intra articular injection within three months or any surgery to the knee joint, patients receiving anticoagulant therapy, patients with hemoglobin less than $10 \mathrm{~g} / \mathrm{dL}$ infection around the knee joint, patients with known thrombocytopenia, and patients with crystal arthropathy or tumor.

The sample size for paired means t-test was calculated using Stata 11 with $85 \%$ power, $5 \%$ alpha, WOMAC mean difference of -13.3 (from a pilot study), standard deviation for WOMAC mean difference of 9.5 (from a pilot study) and estimated drop-out rate of $20 \%$. The minimal required sample size was 81 . From September 2015 to August 2020, 168 primary unilateral knee OA cases met the inclusion criteria and received the single intra-articular injection of PRP. Those patients were assessed with WOMAC and VAS scores before PRP injection, at 3 weeks, 3 months, 6 months and 12 months follow-up. A total of 89 patients who completed the one-year follow were included into this study.

\section{Platelet rich plasma preparation}

The blood (27 $\mathrm{ml}$ of venous blood) sample was extracted in six $6 \mathrm{ml}$ sterile tubes containing $0.7 \mathrm{ml}$ of CPD-A1. The samples were gently shaken to thoroughly mix the anticoagulant with the blood. The blood samples were placed in a centrifuge and centrifuged for 12 minutes at 3500 rpm resulting in the three following layers. The inferior layer was composed of erythrocytes, the intermediate layer consisted of leucocytes with platelets and the superior layer comprised plasma. The superior layer consisting of platelet poor plasma was first discarded. The intermediate buffy coat layer, consisting of platelets mixed with white blood cells was then gently aspirated with an $18 \mathrm{G}$ epidural needle syringe in a volume of $3 \mathrm{ml}$ of PRP and used for intra-articular injection within 30 minutes. An aliquot of product was sent to the laboratory for platelet concentration \& bacteriological examination. All procedures were performed by the researcher at the outpatient department. 
Table 1. Baseline characteristics of the study subjects

\begin{tabular}{l|lc}
\hline Age, mean \pm SD(years) & $57.56 \pm 6.01$ & \\
\hline Sex, M: F & $19: 70$ & \\
\hline BMI, mean \pm SD & $25.92 \pm 3.91$ & \\
\hline Kellgren \& Lawrence grade(K\&L) & Male & Female \\
\hline 1 & 4 & 26 \\
\hline 2 & 6 & 22 \\
\hline 3 & 9 & 22 \\
\hline WOMAC & $47.08 \pm 8.50$ \\
\hline VAS & $69.02 \pm 9.58$ & \\
\hline $\begin{array}{l}\text { Platelet concentration in PRP, } \\
\text { mean } \pm \text { SD }\left(x 10^{3} \text { platelet } / \mathrm{ml}\right)\end{array}$ & $1000.66 \pm 291.91$ & \\
\hline
\end{tabular}

WOMAC; Western Ontario and McMaster Universities Arthritis Index, VAS; visual analog scale, PRP; platelet rich plasma

\section{Treatment procedure and follow-up}

The patient was placed in the supine position with the knee fully extended. Under aseptic condition, $2.5 \mathrm{cc}$ of PRP was injected in the suprapatellar pouch using a superolateral approach with a 22-gauge needle without local anaesthesia. At the end of the procedure, the patient was invited to bend and extend the knee for a few seconds to distribute the PRP itself throughout the joint. The patient was discharged after 30 minutes of observation. They were reassessed with WOMAC score and VAS at the end or 3 weeks, 3 months, 6 months and 12 months after treatment. During the follow-up period, NSAIDs were not allowed and paracetamol $500 \mathrm{mg}$ three times daily was prescribed in case of discomfort. All patients were asked to stop analgesic medications 24 hours before follow-up reassessment.

\section{Data collection and statistical analysis}

Data were collected through pre-structured pro-forma by medical personnel unaware of the procedure. All statistical analyses were performed using Microsoft Excel 2016 (Microsoft Inc., Seattle, WA, USA). A $p$ value of less than 0.05 was considered statistically significant. The WOMAC and VAS scores before and after intervention were compared using paired-sample t-tests. Pearson's correlation coefficient ( $r$ ) and regression analysis were calculated between the platelet concentration in PRP and improved outcomes.

\section{Results}

All patients in this study had a mean age of 57.5 years (range, 50-80), mean BMI of 25.92 (range, 18.45-34.13) and other base line characteristics were reported (Table 1). Among 89 patients with unilateral primary knee OA treated by single injection of PRP, no major adverse events were observed during the procedure and the follow-up period. Statistically significant improvement of all functional and pain scores was observed through-out the follow-up compared with the baseline scores (Table 2). All patients were satisfied with their results.

The mean WOMAC score improved from 47.08 points (range, 28-66) before intervention to 20.37 points (range, 5-45) at 3 weeks, 23.24 points (range, 6-50) at 3 months, 29.89 points (range, 5-60) at 6 months and 27.78 points (range, $5-70)$ at 12 months and final follow-up showing a statistically significant improvement $(p<0.001)$ at each of the follow-ups with respect to baseline (Figure 1). Mean VAS score also improved from 69.02 points (range, 50-85) before intervention to 36.23 points (range, 10-80) at 3 weeks, 37.04 points (range, 10-80) at 3 months, 42.58 points (range, 5-80) at 6 months, 39.15 points (range, 5-75) at 12 months, showing a statistically significant difference $(p<0.001)$ at each of the follow-ups with respect to baseline (Figure 1). 
Table 2. Clinical outcome scores before and after PRP injection

\begin{tabular}{|c|c|c|c|c|c|}
\hline Outcomes & $\begin{array}{c}\text { Baseline } \\
\text { Mean } \pm \text { SD }\end{array}$ & $\begin{array}{c}3 \text { weeks } \\
\text { Mean } \pm \text { SD } \\
p \text {-value }\end{array}$ & $\begin{array}{c}3 \text { months } \\
\text { Mean } \pm \text { SD } \\
p \text {-value }\end{array}$ & $\begin{array}{c}6 \text { months } \\
\text { Mean } \pm \text { SD } \\
p \text {-value }\end{array}$ & $\begin{array}{c}12 \text { months } \\
\text { Mean } \pm \mathrm{SD} \\
p \text {-value }\end{array}$ \\
\hline WOMAC & $47.08 \pm 8.50$ & $\begin{array}{c}20.37 \pm 10.09 \\
\quad<0.001\end{array}$ & $\begin{array}{c}23.24 \pm 11.39 \\
<0.001\end{array}$ & $\begin{array}{c}29.89 \pm 14.95 \\
\quad<0.001\end{array}$ & $\begin{array}{c}27.78 \pm 16.56 \\
<0.001\end{array}$ \\
\hline VAS & $69.02 \pm 9.58$ & $\begin{array}{c}36.23 \pm 15.72 \\
\quad<0.001\end{array}$ & $\begin{array}{c}37.04 \pm 17.30 \\
<0.001\end{array}$ & $\begin{array}{l}42.58 \pm 22.15 \\
\quad<0.001\end{array}$ & $\begin{array}{c}39.15 \pm 23.93 \\
<0.001\end{array}$ \\
\hline
\end{tabular}

WOMAC; Western Ontario and McMaster Universities Arthritis Index, VAS; visual analog scale $p<0.001$ (compared to baseline score)

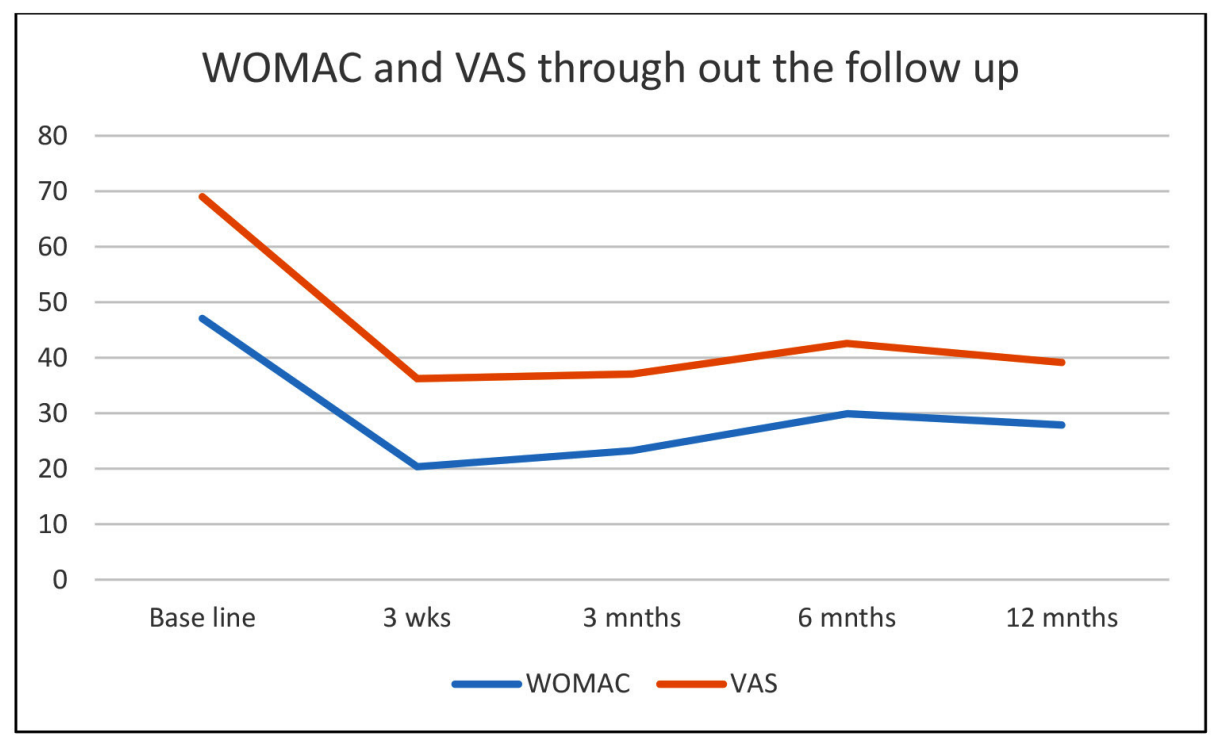

Figure 1. Overall assessment with VAS and WOMAC scores before injection and through to the follow-up period

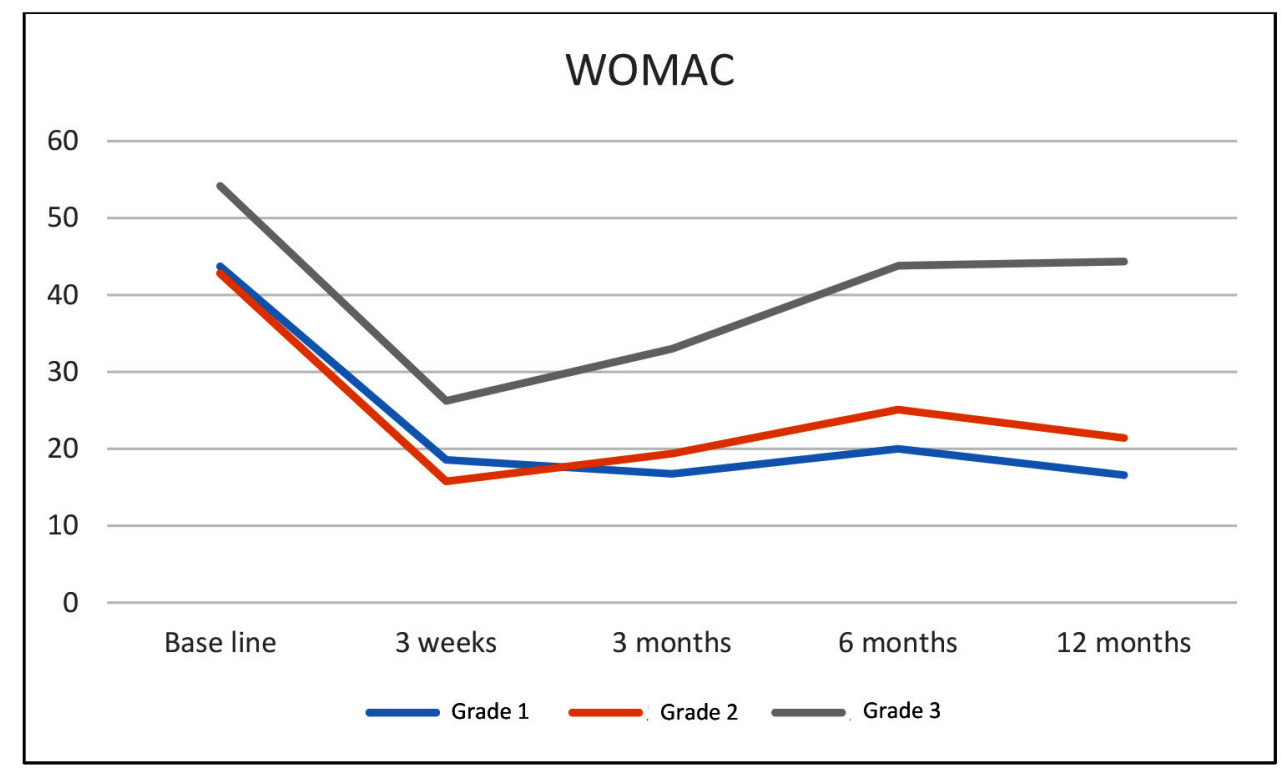

Figure 2. Comparison of WOMAC scores, All the stages improved significantly more in terms of WOMAC at 3 weeks compared with the baseline but the difference of improvement declined at 6 months postintervention. At 12 months after intervention, the scores of both grades 1 and grade 2 were significantly better than those of grade 3 . 


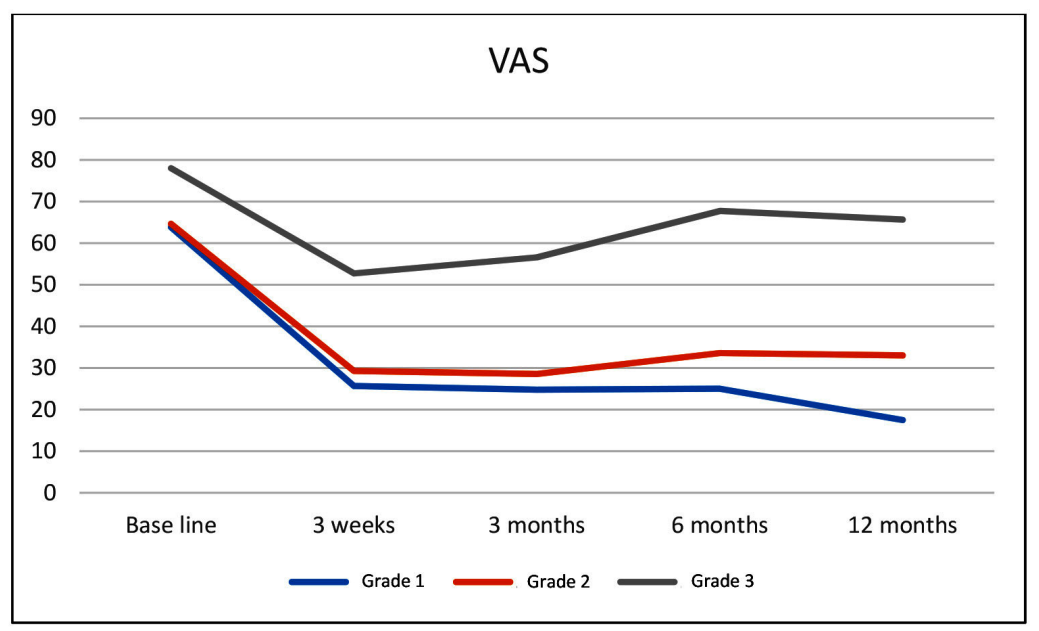

Figure 3. Comparison of VAS scores, All the grades improved significantly more in terms of VAS at 3 weeks compared with the baseline but the difference in improvement declined at 3 months postintervention in grades 2 and 3. At 12 months after intervention, the scores of grade 1 were better than those of 3 weeks whereas in grades 2 and 3, both the improvement pain scores decreased more than those at 3 weeks but were still better than baseline.

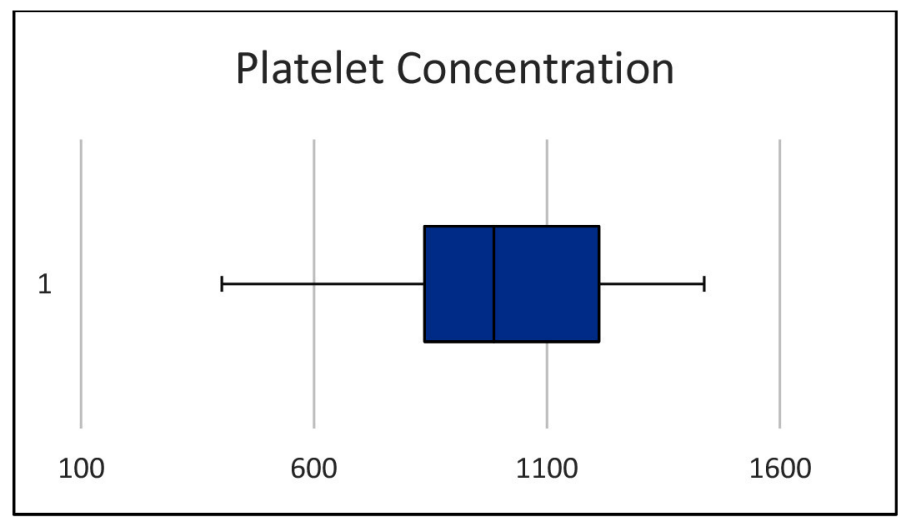

Figure 4. Box plot showing variability of the concentration of platelet in PRP

According to the K\&L grade of knee OA, all grades in this study improved significantly in terms of WOMAC score at 3 weeks compared with that of baseline but the difference of improvement declined at 6 months postintervention. At 12 months after intervention, the WOMAC scores of both grades 1 and 2 were better than those of grade 3 (Figure 2). Regarding VAS, all grades significantly improved at 3 weeks compared with the baseline but the difference in improvement declined at 3 months postintervention in grades 2 and 3 . In the grade 1 OA knee group, the VAS scores at 12 months were better than those at 3 weeks whereas in grades 2 and 3, both the improved pain scores decreased more than those at 3 weeks but were still better than baseline (Figure 3).

The platelet concentration was evaluated in every case of PRP injection. A high variability in platelet concentration in PRP was found. We observed that the mean concentration of platelet in PRP was $1000.66 \times 10^{3}$ platelets $/ \mathrm{mL}$ with a range of $402 \times 10^{3}$ platelets $/ \mathrm{mL}$ to $1630 \times 10^{3}$ platelets/ $\mathrm{mL}$ without outlier (Figure 4). Average platelet concentration yielded 5.2 fold more than baseline. Correlation between the mean improvement scores (difference between each follow up score and the baseline) of both WOMAC and VAS with platelet concentration was determined (Table 3). Although positive correlations were found between platelet concentration and mean improvement scores of WOMAC and VAS at 3 weeks $(r=0.31$, $\mathrm{r}=0.40), 3$ months $(\mathrm{r}=0.10, \mathrm{r}=0.23), 6$ months $(\mathrm{r}=0.08, \mathrm{r}=0.30)$ and 12 months at final follow-up $(\mathrm{r}=0.12, \mathrm{r}=0.23)$, the most significant results were seen at 3 weeks postintervention (Figures 5, 6). 
Table 3. Correlation coefficient ( $\mathrm{r}$ ) between clinical improvement scores and platelet concentration

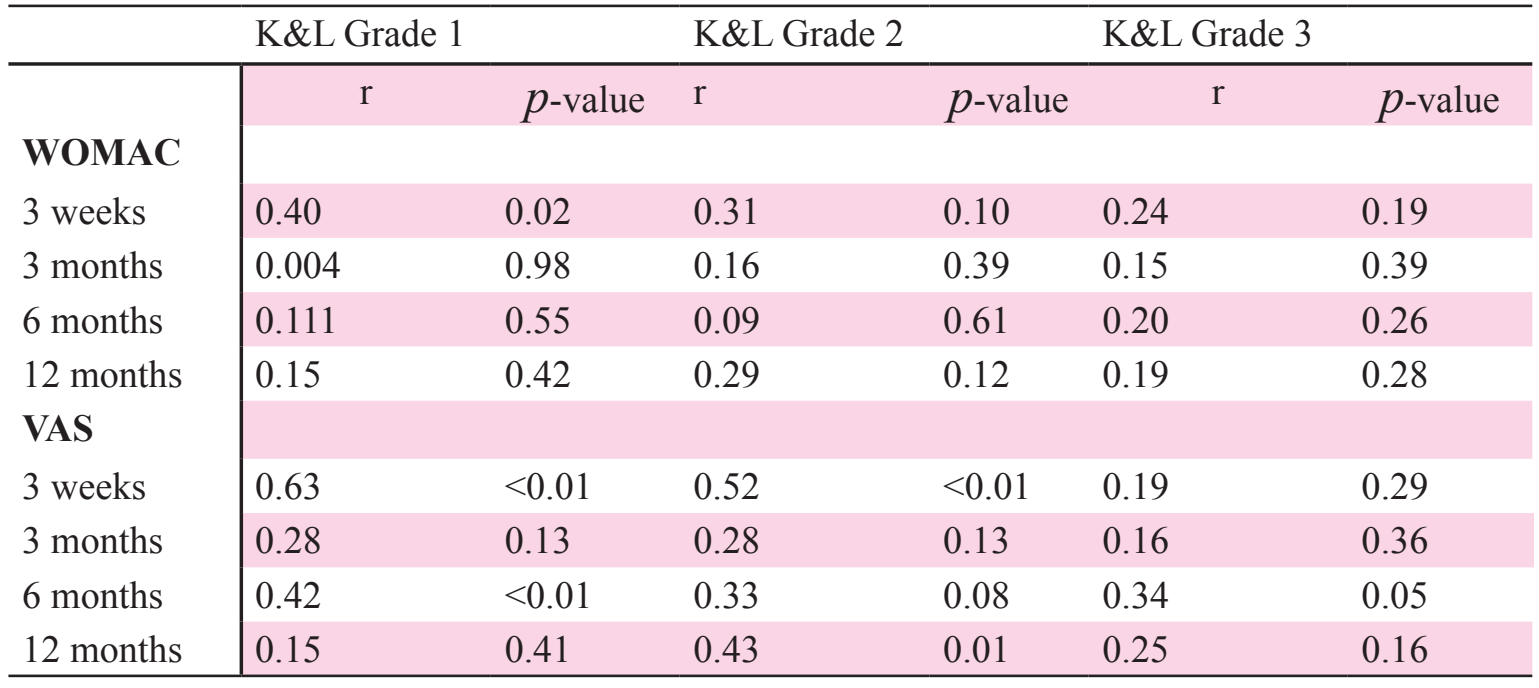

WOMAC; Western Ontario and McMaster Universities Arthritis Index, VAS; visual analog scale, K\&L; Kellgren \& Lawrence Radiographic Knee OA classification

WOMAC 3 weeks improvement scores correlates the PLT count

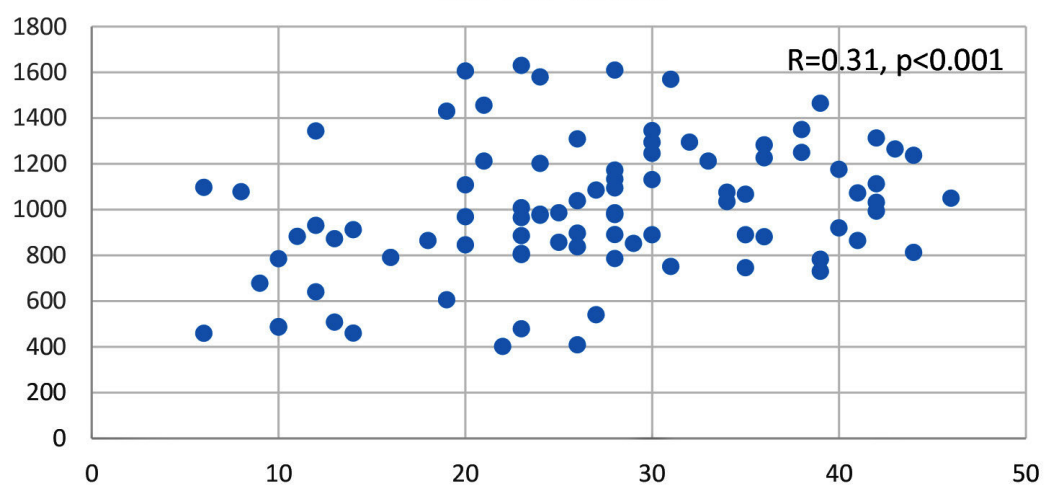

Figure 5. Correlation between improvement in WOMAC at 3 weeks and platelet concentration, WOMAC improvement on the $\mathrm{X}$-axis and concentration of the platelet was plotted on the Y-axis.

VAS 3 weeks improvement correlates with PLT count in PRP

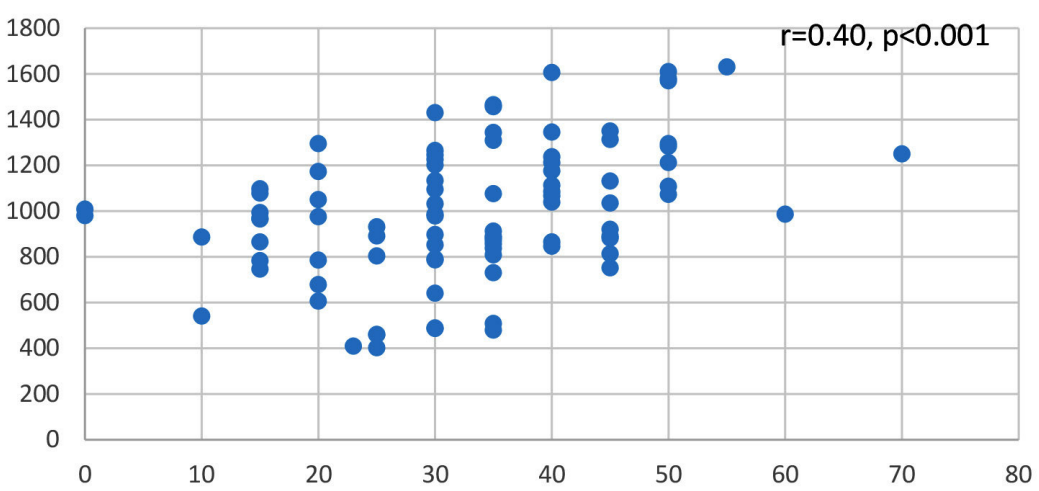

Figure 6. Correlation between improvement in VAS at 3 weeks and platelet concentration, VAS improvement on the $\mathrm{X}$-axis and concentration of the platelet was plotted on the Y-axis. 
Table 4. Clinical improvement scores and platelet concentration

\begin{tabular}{|c|c|c|c|}
\hline & PLT $<837 \times 10^{3} / \mathrm{mL}$ & PLT $>1212 \times 10^{3} / \mathrm{mL}$ & $p$-value \\
\hline K\&L grade 1 & 10 & 9 & \\
\hline K\&L grade 2 & 6 & 7 & \\
\hline K\&L grade 3 & 5 & 5 & \\
\hline 3 weeks WOMAC (Mean \pm SD) improvement & $22.38 \pm 10.87$ & $30.57 \pm 8.71$ & 0.01 \\
\hline 12 months WOMAC (Mean \pm SD) improvement & $22.5 \pm 12.13$ & $23.69 \pm 9.82$ & 0.7 \\
\hline 3 weeks VAS (Mean \pm SD) improvement & $27.76 \pm 9.06$ & $41.52 \pm 10.91$ & 0.001 \\
\hline 12 months VAS $($ Mean \pm SD) improvement & $30.9 \pm 19.49$ & $40.65 \pm 20.90$ & 0.1 \\
\hline
\end{tabular}

WOMAC; Western Ontario and McMaster Universities Arthritis Index, VAS; visual analog scale

According to platelet concentration, patients were divided in two groups: patients with a platelet count within the first quartile (platelet count $<837 \times 10^{3} / \mathrm{mL}$ ) and those with a platelet count of more than the third quartile (platelet count $>1212 \times 10^{3} / \mathrm{mL}$ ) (Table 4). Each group consisted of 21 patients. Mean improvement score of WOMAC at 3 weeks in the lower platelet group was $22.85 \pm 10.87$ and that in the higher group was $30.26 \pm 8.56$ showing a statistically significant difference $(p=0.01)$ whereas mean WOMAC improvement score at 12 months in the lower platelet group was $22.5 \pm 12.13$ and that in the higher group was $23.69 \pm 9.82$ showing no difference $(p=0.7)$. Similar results were observed in VAS pain score. Mean improvement score of the VAS at 3 weeks in the lower platelet group was $28.9 \pm 8.42$ and that in the higher group was $41.52 \pm 10.91$ showing a statistically significant difference $(p=0.001)$. However, mean VAS improvement score at 12 months in the lower platelet group was $30.9 \pm 19.49$ and that in the higher group was $40.65 \pm 20.90$ showing no difference $(p=0.1)$. The outcome scores in patients receiving PRP with a higher concentration of platelet were better than those at lower concentrations especially at 3 weeks. Nevertheless, this relationship was negligible in the later follow-up visits irrespective of osteoarthritis severity.

\section{Discussion}

This study aimed to determine whether the platelet concentration in PRP influenced the outcome of primary OA knee. Several studies had proved that platelet rich plasma had posi- tive effects on ligamentous injury, tendinopathy and cartilage lesions and knee OA. ${ }^{(22-26)}$ Various factors may impact the clinical outcome of PRP injection in primary knee OA such as the platelet concentration in PRP, the doses and frequency of injection as well as the preparation method of PRP.

Theoretically, the platelet concentration may affect the outcome of the disease process. To our knowledge, limited scientific evidence is available regarding optimal platelet concentration to treat knee OA. Patel et al. reported that a single injection of PRP containing 10 times the baseline platelet count was as effective as injecting twice at platelet concentration of more than 4 times baseline. ${ }^{(6)}$ However, the optimal concentration of platelet in PRP to obtain the maximal result was unreported. However, the study of Bahar et al. stated that the concentration of $1,000 \times 10^{3}$ platelet $/ \mathrm{mL}$ was sufficient for pain relief and functional recovery in treating early OA. ${ }^{(21)}$ Whether the concentration of less than $1,000 \times 10^{3}$ platelet $/ \mathrm{mL}$ influenced the outcomes remained unconfirmed. In the present study, the maximal relieving effect of PRP yields at 3 weeks postintervention irrespective of platelet concentration and the relieving effect seemed to be sustained until 12 months comparable to the study of Kon et al. ${ }^{(7)}$ Moreover, the positive correlation between the improvement scores of WOMAC and VAS and the platelet concentration in the PRP, was found regardless of the severity of OA of the patients. The highest improvement outcome scores were observed only at 3 weeks after intervention and the improvement scores gradually declined throughout the later follow-ups. These might 
have been the reasons for the life span of platelets and the gradual increase in the release of growth factors up until the $19^{\text {th }}$ day and the consecutively delivered slow and constant release until the $23^{\text {th }}$ day. ${ }^{(27)}$

Many studies have suggested the clinical application of PRP via multiple injections to favor knee OA healing at 3 weeks, once monthly or 3 months. ${ }^{(6,13)}$ In our study, we used single injection of PRP in OA knee and observed that PRP injection produced favorable outcomes. This effect could be sustained over one year whereas in the study of Filardo et al., the mean beneficial effect of lasted ten months with sustained action up to 24 months. ${ }^{(24)}$ In the study of Patel et al., single injection of PRP also produced comparable results as injecting twice. ${ }^{(6)}$

Different PRP separation systems and devices were used (Arthrex ACP Double Syringe System and Biomet Biologics GPS System). Briefly, they relied on single centrifugation, double centrifugation and onmanual or automatic systems operated in open or closed circuits with platelet concentrations varying from 1.99 to 9.3 fold over baseline. (6, 28-30) However, no standardized preparation methods and optimal concentration of platelets in PRP that induce maximal pain relief remain unknown. ${ }^{30-32)}$

Diverse PRP preparation and application techniques of PRP have been used for platelet activation and inactivation. Both PRP formulations provide pain relief and none is more efficacious than the other. ${ }^{(33)}$ In this study, we used an inactivated technique which also produced quite relieving effects on OA knee pain.

In the present study, centrifugation procedures to prepare PRP increased the platelet count as well as white blood cell concentration. However, related studies have already pointed out the key role of leucocytes in PRP for their anti-infectious action, immune regulation and potential regeneration effects. ${ }^{(34,35)}$ The leucocyte content did not seem to induce negative effects or impair the potential beneficial effects of PRP, even when used in the joints. However, the study of Milants et al. recommended using a single spinning technique, a platelet concentration of lower than 5 times baseline and avoidance of leukocytes. ${ }^{(36)}$
The present study showed that increased white blood cells in PRP produced no negative effects on the knee joint.

In this study, no major adverse events related to the injections were observed during the treatment and follow-up periods. Some authors have reported some injection pain, local inflammation of short duration, and re-accumulation of effusion, but these symptoms resolved spontaneously. ${ }^{(6,24,26)}$ In the present study, all the functional and pain scores improved and were maintained until 12 months compared with baseline scores irrespective of platelet count in PRP. Although PRP has affected both functional and pain scores of patients up to one year, the influence of platelet concentration was observed only at early postintervention.

This study constitutes one of the first in vivo studies in our hospital to treat knee OA with PRP injection. However, it encountered some limitations. We evaluated the platelet count in PRP but did not include red and white blood cells. We didn't use commercial platelet concentration systems, the platelet concentration was not homogeneous and revealed high variability in this study. Moreover, the drop-out rate in this study was very high because the patients from the outreach area were challenged to complete the one year follow-up visit. Despite these limitations, this study demonstrated that PRP is a potentially safe, simple and low cost method to improve articular joint healing, with promising results in treating early stage primary knee OA.

\section{Conclusion}

A significant positive correlation was observed between the improvement of clinical outcomes and platelet concentration in PRP especially at 3 weeks postintervention suggesting repeating injection might be beneficial. Further study would also be required to compare different PRP formulations and preparation methods either manually or via the use of commercial kits.

\section{Conflict of interest}

The authors declare they have no conflict of interest. 


\section{Acknowledgement}

We thank Associate Professor Dr. Thidar Win, Department of Preventive and Social Medicine, Mandalay, Myanmar for statistical support in this study.

\section{REFERENCES}

1. Laudy AB, Bakker EW, Rekers M, Moen $\mathrm{MH}$. Efficacy of platelet-rich plasma injections in osteoarthritis of the knee: a systematic review and meta- analysis. Br J Sports Med 2015; 49: 657-72.

2. Anitua E, Sanchez M, Javier Aguirre JJ, Prado R, Padilla S, Orive G. Efficacy and safety of plasma rich in growth factors intra-articular infiltrations in the treatment of knee osteoarthritis. Arthroscopy 2014; 30: 1006-17.

3. Shen L, Yuan T, Chen S, Xie X, Zhang C. The temporal effect of platelet-rich plasma on pain and physical function in the treatment of knee osteoarthritis: systematic review and meta-analysis of randomized controlled trials. J Orthop Surg Res 2017; 12: 16.

4. Meheux CJ, McCulloch PC, Lintner DM, Varner KE, Harris JD. Efficacy of intraarticular platelet-rich plasma injections in knee osteoarthritis: a systematic review. Arthroscopy 2016; 32: 495-505.

5. Chang KV, Hung CY, Aliwarga F, Wang TG, Han DS, Chen WS. Comparative effectiveness of platelet-rich plasma injections for treating knee joint cartilage degenerative pathology: a systematic review and meta-analysis. Arch Phys Med Rehabil 2014; 95: 562-75.

6. Patel S, Dhillon M. S, Aggarwal S, Marwaha $\mathrm{N}$, Jain A. Treatment of platelet rich plasma is more effective than placebo for knee osteoarthritis. Am J Sports Med. 2013; 41: 356-64.

7. Kon E, Buda R, Filardo G, Martino AD, Timoncini A, Cenacchi A, et al. Platelet-rich plasma intraarticular knee injections produced favourable results on degenerative cartilage lesions. Knee Surg Sports Traumatol Arthrosc 2010; 18: 472-9.

8. Sampson S, Gerhardt M, Mandelbaum B. Platelet rich plasma injection grafts for musculoskeletal injuries. Curr Rev Musculoskelet Med 2008; 1:165-74.
9. Nurden AT. Platelets, inflammation and tissue regeneration: Thrombosis \& Haemostasis Supplement. Schattauer 2011; S13-S33.

10. Dhillon RS, Schwarz EM, Maloney MD. Platelet-rich plasma therapy-future or trend? Arthritis Res Ther 2012; 14: 219.

11. Hickey DG, Frenkel SR, Di Cesare PE. Clinical applications of growth factors for articular cartilage repair. Am J Orthop 2003; 32: 70-6.

12. Dhurat R, Sukesh M. Principles and methods of preparation of platelet- rich plasma: A review and author's perspective. J Cutan Aesthet Surg 2014; 7: 189-97.

13. Spakova T, Rosocha J, Lacko M, Harvanova D, Gharaibeh A. Treatment of knee joint osteoarthritis with autologous platelet rich plasma in comparison with hyaluronic acid. Am J Phys Med Rehabi 2012; 91: 1-7.

14. Magalon J, Bausset O, Serratrice N, Giraudo L, Aboudou H, Veran J, et al. Characterization and comparison of 5 platelet- rich plasma preparations in a single-donor model. Arthroscopy 2014; 30: 629-38.

15. Sundman EA, Cole BJ, Fortier LA. Growth factor and catabolic cytokine concentrations are influenced by the cellular composition of platelet-rich plasma. Am J Sports Med 2011; 39: 2135-140.

16. Magalon J, Bausset O, Serratrice N, Giraudo L, Adoudou H, Veran J, et al. Characterization and comparison of 5 platelet- rich plasma preparations in a single-donor model. Arthroscopy 2014; 30: 629-38.

17. Sundman EA, Cole BJ, Fortier LA. Growth factor and catabolic cytokine concentrations are influenced by the cellular composition of platelet-rich plasma. Am J Sports Med 2011; 39: 2135-40.

18. Castillo TN, Pouliot MA, Kim HJ, Dragoo JL. Comparison of growth factor and platelet concentration from commercial platelet- rich plasma separation systems. Am J Sports Med 2011; 39: 266.

19. Eppley BL, Woodell JE, Higgins J. Platelet quantification and growth factor analysis from platelet-rich plasma: implications for wound healing. Plast Reconstr Surg 2004; 114: $1502-8$. 
20. Weibrich G, Hansen T, Kleis W, Buch R, Hitzler WE. Effect of platelet concentration in platelet-rich plasma on peri-implant bone regeneration. Bone 2004; 34: 665-71.

21. Dernek B, Kesiktas FM, Duymus TM, Aydin T, Isiksacan N, Diracoglu D, et al. Effect of platelet concentration on clinical improvement in treatment of early stage- knee osteoarthritis with platelet-rich plasma concentrations. J Phys Ther Sci 2017; 29: 896-901.

22. Aqil A, Jacobs L. Physical therapy or platelet rich plasma injections in the treatment of tennis elbow: A ramdomised clinical trial. Webmed Central ORTHOPAEDICS 2010; 1: WMC00701

23. Engebretsen L, Schamasch P. The use of platelet-rich plasma in sports medicine-The international olympic committee opinion. Operat Tech Orthop 2012; 22: 43-8.

24. Filardo G, Kon E, Buda R, Timoncini A, Martino AD, Cenacchi A, et al. Platelet-rich plasma intra articular knee injections for the treatment of degenerative cartilage lesions and osteoarthritis. Knee Surg Sports Traumatol Arthrosc 2011; 19: 528-35.

25. Andia I, Sanchez M, Maffulli N. Joint pathology $\&$ platelet rich plasma therapies. Expert Opin Biol Ther 2012; 12: 7-22.

26. Sánchez M, Fiz N, Azofra J, Usabiaga J, Recalde EA, Gutierrez AG, et al. A randomized clinical trial evaluating plasma rich in growth factors (PRGF Endoret) versus hyaluronic acid in the ahort-term treatment of symptomatic knee osteoarthritis. Arthroscopy 2012; 28: 1070-8.

27. Chatterjee A, Debnath K. Comparative evaluation of growth factors from platelet concentrates: An in vitro study. J Indian Soc Periodontol 2019; 23: 322-8.

28. Gato-Calvo L, Magalhaes J, Ruiz- Romero C, Blanco FJ, Burguera EF. Platelet- rich plasma in osteoarthritis treatment: review of current evidence. Ther Adv Chronic Dis 2019; 10: $1-18$.

29. Machado ES, Leite R, Santos CCd. Artuso GL, Gluszczak F, Jesus LGD, et.al. Turn down-turn up: a simple and low- cost protocol for preparing platelet-rich plasma. CLINICS 2019; 74: e1132

30. Everts PAM, Mahoney CB Hoffmann JJML, Schönberger JPAM, Box HAM, Zundert AV, et al. Platelet-rich plasma preparation using three devices: implications for platelet activation and platelet growth factor release. Growth Factors 2006; 24: 165-71.

31. Kevy SV, Jacobson MS. Comparison of methods for point of care preparation of autologous platelet gel. J Extra Corpor Technol 2004; 36: 28-35.

32. Tamimi FM, Montalvo S, Tresguerres I, Blanco Jerez L. A comparative study of 2 methods for obtaining platelet-rich plasma. J Oral Maxillofac Surg 2007; 65:1084-93.

33. Kuffler DP. Variables affecting the potential efficacy of PRP in providing chronic pain relief. J Pain Res 2019; 12: 109-16.

34. Bielecka AC, Ehrenfest DMD, Lubkowska A, Bielecki T. Microbicidal properties of leukocyte-and platelet-rich plasma/fibrin (L-PRP/L-PRF): New perspectives. J Biol Regul Homeost Agents 2012; 26 (2 Suppl 1): 43S-52S.

35. Lana JF, Macedo A, Ingrao ILG, Huber SC, Santos GS, Santana MHA. Leukocyte-rich PRP for knee osteoarthritis: Current concepts. J Clin Orthop Trauma 2019: 10 (Suppl 1): S179-S82.

36. Milants C, Bruyere O, Kaux JF. Responders to platelet-rich plasma in osteoarthritis: A technical analysis. Biomed Res Int 2017: 7538604 . 